\title{
Intraprosthetic dislocation after a revision hip replacement: a case report
}

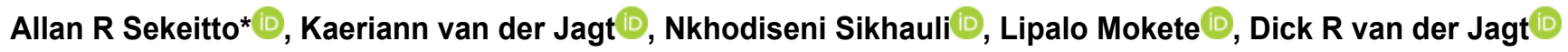 \\ Arthroplasty Unit, Division of Orthopaedic Surgery, Charlotte Maxeke Johannesburg Academic Hospital, University of the Witwatersrand, Johannesburg,
} South Africa

*Corresponding author: sekeitto@yahoo.com

Citation: Sekeitto AR, Van der Jagt K, Sikhauli N, Mokete L, Van der Jagt DR. Intraprosthetic dislocation after a revision hip replacement: a case report. SA Orthop J 2021;20(1):49-52. http://dx.doi.org/10.17159/23098309/2021/v20n1a7

Editor: Dr Chris Snyckers, University of Pretoria, Pretoria, South Africa

Received: May 2020

Accepted: September 2020

Published: March 2021

Copyright: @ 2021 Sekeitto AR. This is an open-access article distributed under the terms of the Creative Commons Attribution Licence, which permits unrestricted use, distribution and reproduction in any medium, provided the original author and source are credited.

Funding: No benefits in any form have been received or will be received from a commercial party related directly or indirectly to the subject of this article.

Conflict of interest: DVJ reports consultancy for Smith \& Nephew, all outside the submitted work. LM reports consultancy for Zimmerbiomet and Implantcast; payment for lectures including service on speakers' bureaus for Zimmerbiomet, Smith \& Nephew and Advanced Orthopaedics, all outside the submitted work. The other authors declare no conflict of interest relevant to this work.

\begin{abstract}
\section{Background}

The dual mobility cup (DMC) was initially design in 1974. It was designed to offer additional stability in total hip arthroplasty (THA) and to prevent dislocations. The dissociation of a DMC has been termed an intraprosthetic dislocation (IPD) and is a rare complication. It is defined as separation of the articulation between the polyethylene and head articulation in the DMC. As the utilisation of DMCs in orthopaedic surgery increases, we can expect an increase in this rare complication. We report a case of an IPD in the setting of revision hip arthroplasty in a 72-yearold female.
\end{abstract}

\section{Case report}

The report is on a 72-year-old female patient who underwent revision hip arthroplasty. The articulation utilised was of the dual mobility type. Some eight months later she dislocated her hip. An attempted closed reduction under general anaesthesia with muscle relaxant was unsuccessful. Thereafter she was taken to surgery to perform an open reduction of the hip. Intra-operatively it was found that the dual mobility head had dissociated, with the polyethylene component remaining in the metal liner. A revision of the components was performed.

\section{Discussion}

We postulate on the mechanisms of dissociation of the dual mobility head. We review the current literature related to IPD and discuss the risk factors associated with this rare complication.

\section{Conclusion}

The diagnosis of IPD is an indication for revision surgery of the DMC. When utilising a DMC, care should be taken to mitigate against the known risk factors for IPD. All dual mobility dislocations should be reduced under general anaesthesia with muscle relaxant.

Level of evidence: Level 4

Keywords: intraprosthetic dislocation, dual mobility cup, revision hip arthroplasty

\section{Introduction}

The dual mobility cup (DMC) was initially designed in 1974 by Bousquet to offer additional stability to the standard total hip arthroplasty (THA) and prevent dislocations. ${ }^{1-3}$ Instability in THA is a common indication for revision. ${ }^{3-5}$ The literature reports that 17.3 to $22.5 \%$ revisions in THA are performed for instability. ${ }^{6-8}$ The DMC has reduced post-operative dislocation rates in both primary and revision THA. ${ }^{9}$
The risk factors for instability include patients older than 75 years, prior hip surgery, neuromuscular disease, dysplastic hips, spinal abnormalities, ligamentous laxity, small anatomy ${ }^{3,5}$ and neck of femur fractures. The incidence of instability with primary THA is up to $7 \%$ and increases up to $28 \%$ in revision THA. ${ }^{4,10}$ In addition to a DMC, the stability of THA can be further improved by technique modifications such as anterior approach, repair of the posterior soft tissue, increased offset, larger femoral head and increased abductor tension. ${ }^{1}$ 


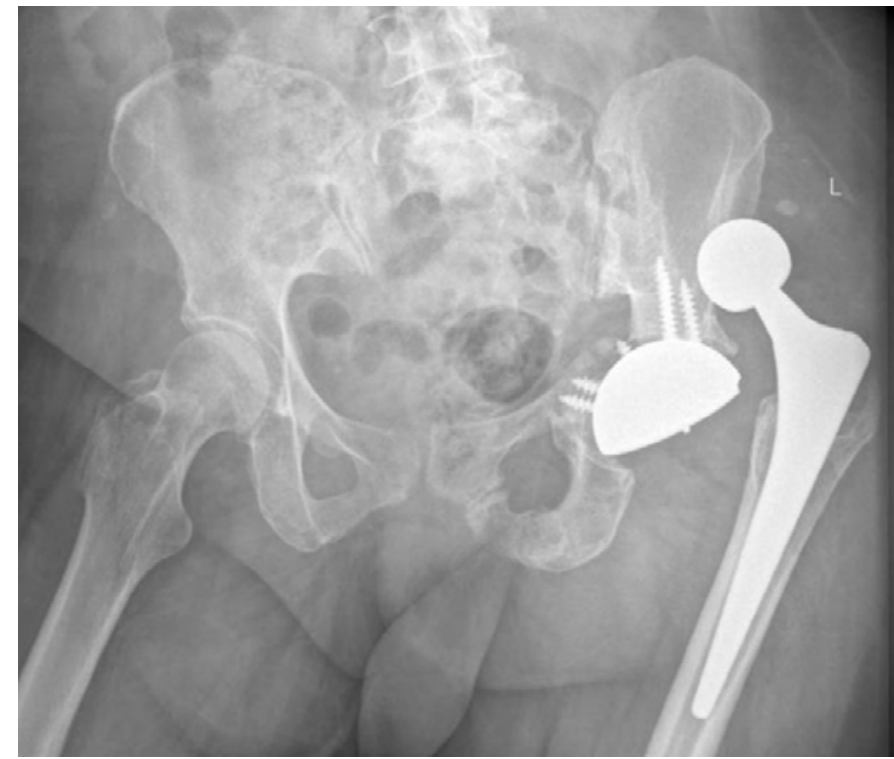

Figure 1. AP radiograph of the pelvis demonstrating the dislocated left hip prosthesis

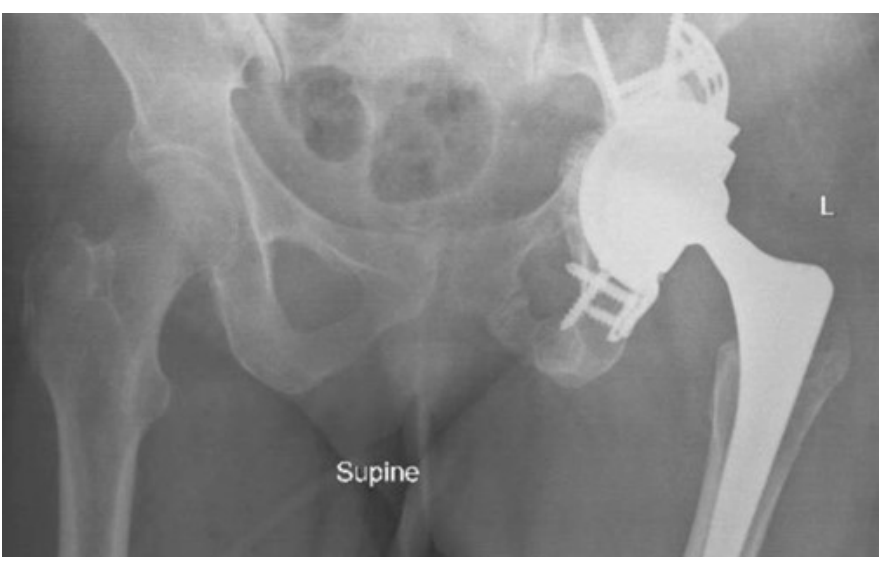

Figure 2. Pelvis AP radiograph demonstrating the revision total hip replacement with the dual mobility cup cemented into the cage

We present a case study of a patient with a dual mobility THA which had a dissociation of the dual mobility head.

\section{Case}

A 72-year-old female patient had undergone five previous hip revisions. The indications for the previous revisions were polyethylene wear and loosening, a poorly fixed femoral component and malposition of the acetabular cup that led to instability. At the final revision, a cup-cage technique was used with a dualmobility component cemented into the cage due to pre-operative instability and pelvic dissociation (Figure 1). The femoral stem was retained, and after the appropriate dual-mobility head was fitted, the reduction was stable (Figure 2). She suffered from ongoing mobility issues associated with severe backache due to underlying thoraco-lumbar kyphoscoliosis. Eight months later she dislocated her hip after a fall. In the X-ray, the lucency of the polyethylene component around the head is visible, indicating dislocation of the dual mobility head (Figure 3). The attempted closed reduction was performed under general anaesthesia with full paralysis; however, it was not successful.

At the open reduction, it was found that head had dissociated, with the polyethylene component found within the metal liner indicating an intraprosthetic dislocation (IPD) (Figure 4). All incisions were through the same scar. The approach used in all the procedures

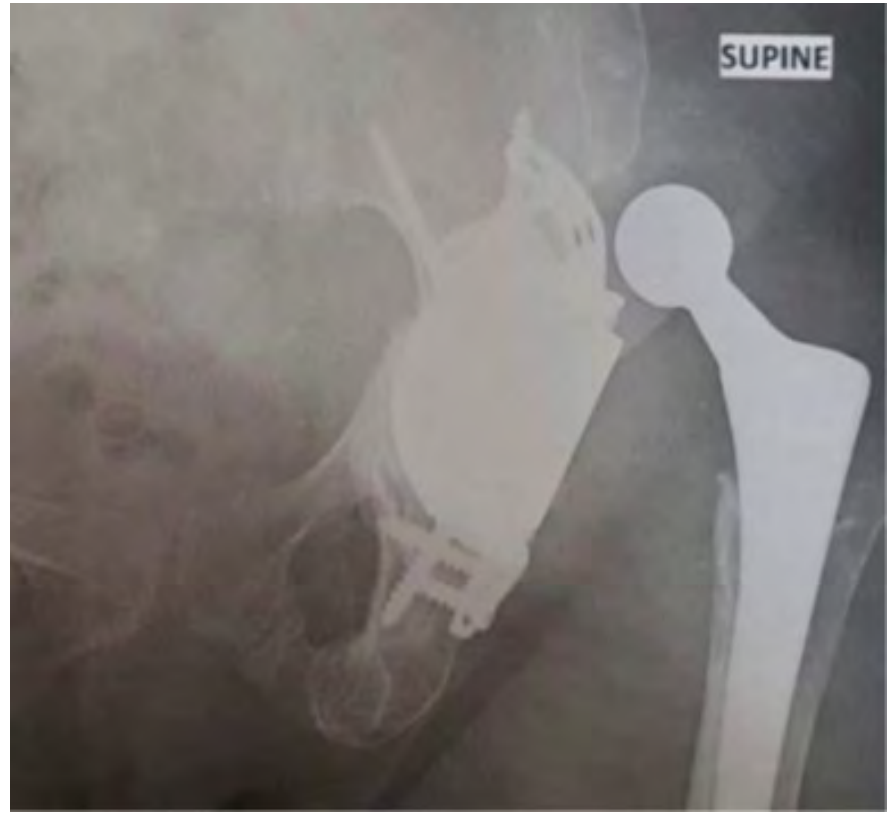

Figure 3. AP radiograph of the left hemipelvis demonstrating the dislocated left hip prosthesis

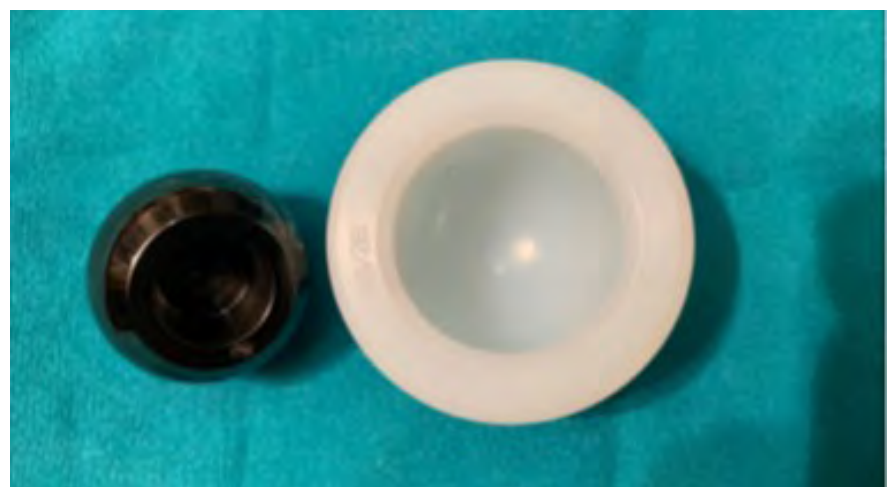

Figure 4. The dissociated components of the dual mobility head

was an antero-lateral type approach. The soft tissues were severely scarred and fibrotic. The contracted fibrotic peri-articular scar tissues held the polyethylene component in the metal shell. We propose the mechanism of IPD was due to the impingement of the polyethylene component on the soft tissues preventing its mobility and resulting in the levering out of the head during the attempted reduction. The same DMC construct was used but with a longer head to increase the abductor tissue tension. There have been no subsequent dislocations.

\section{Discussion}

The dissociation of a DMC has been termed an intraprosthetic dislocation (IPD) or retentive failure ${ }^{11}$ and is a rare complication. It is defined as separation of the articulation between the polyethylene and head articulation, or loss of the polyethylene protective rim and escape of the femoral head from the polyethylene liner, ${ }^{1-3}$ or dissociation of the polyethylene component from the femoral head. ${ }^{9}$ The literature indicates an incidence of $1.9 \%$ to $5.2 \%$ in older generation designs. ${ }^{3}$ The older generation designs failed due to progressive wear of the capture mechanism leading to the IPD. ${ }^{11,12}$ IPD is a late complication occurring eight to 11 years post-operatively with conventional polyethylene. ${ }^{9}$ The current dual mobility designs have an IPD incidence of $0 \%$ to $2.4 \%$ with a followup period of six to nine years. ${ }^{3}$ The current designs lack a capture 
mechanism, with the head being press-fitted in the polyethylene component. De Martino et al. ${ }^{9}$ in their systematic review found 19 early (within nine months) IPD in modern DMC designs. IPD cannot be reduced closed and requires operative management, with change of modular head and liner or revision of the acetabular cup. ${ }^{9,11}$ Tabori-Jensen et al. ${ }^{13}$ in their cohort of 966 DMCs observed eight IPD, six of which were related to an attempted closed reduction of dislocation. All required revision surgery with liner and femoral head replacement. They had 45 DMC dislocations and found that increased cup inclination $(p=0.04)$ and cup retroversion $(p<0.001)$ were risk factors in the dislocation group. Although it has been suggested, DMCs are not limited to the Lewinnek safe zones. ${ }^{14}$ Addona et al. ${ }^{15}$ reported five cases of IPD in their single surgeon case series, all of which were secondary to an attempted closed reduction of a DMC dislocation. In addition, Rotini et al. ${ }^{16}$ reported on two cases of IPD secondary to an attempted closed reduction. The review of the literature makes our case the 35th early IPD and 31st secondary to an attempted closed reduction of a dislocated DMC.

\section{Biomechanics}

The principle of the DMC is the smaller inner femoral head which is in keeping with Charnley's low friction arthroplasty and the larger unconstrained polyethylene component within the cup. ${ }^{13}$ The dual mobility design has two articulating surfaces. The primary articulation takes place between the femoral head and the polyethylene component during activities of daily living (ADL). ${ }^{3}$ The secondary articulation occurs between the polyethylene component and the acetabular shell, and is involved in activities beyond the usual ADL which involve extremes of motion, resulting in contact of the neck of the femoral stem and rim of the polyethylene component, ${ }^{3}$ and allowing the secondary articulation to extend the range of movement. The design allows for a greater arc of motion, ${ }^{1}$ increased 'jump distance' and increased head-to-neck ratio which increases stability (McKee-Farrar principle), ${ }^{17}$ and resulting reduced dislocation/subluxation risk. . $^{3,418}$

\section{Modern designs}

The complication of IPD has been addressed with modern designs. Modern design modifications include: smooth neck surfaces, skirts on the femur, retentive rim modifications to press-fitted head, increased tolerances of external and internal diameters, modern polyethylene and alterations in fixation surfaces, cup configuration and femoral neck design. ${ }^{1}$ Philippot and colleagues found in their study report of late IPD that the most common cause was secondary play of the inner head due to polyethylene wear. ${ }^{2}$ The use of highly crosslinked polyethylene has decreased wear and subsequent IPD secondary to play of the inner head..$^{3,9}$ The use of skirted femoral heads has been recommended against. The risk is earlier neck-cup impingement in the arc of motion due to reduction of the head-to-neck ratio and increasing the potential dislocation risk. ${ }^{9,14}$ The available literature that was reviewed shows no cases of early IPD occurring in patients with a femoral head smaller than $28 \mathrm{~mm}$ or in patients in which the DMC was used in conjunction with a skirted femoral head. ${ }^{9}$ Chouteau et al. ${ }^{19}$ reported no cases of IPD in their cohort of 240 fourth-generation DMCs at $7-$ to 11-year follow-up, highlighting the rarity of the complication in modern designs and ultra-high molecular weight polyethylene. The potential for polyethylene wear with subsequent IPD still exists and may present several years after implantation. ${ }^{20}$

\section{Component pairing}

The literature reports off-label practice of mixing components from different manufacturers at revision procedures to minimise complications in removing well-fixed components, if the components are appropriately sized..$^{21}$ De Martino et al. ${ }^{9}$ indicated within their reported cases only six reported cases (32\%) of early IPD occurring in patients with a femoral head and polyethylene liner manufacturers' mismatch. There were a further nine cases $(47 \%)$ in patients with no mismatch, and in four cases $(21 \%)$ the components were not specified. ${ }^{9}$ Component pairing is a suspected risk factor for IPD. ${ }^{9}$ In our case the components were matched.

\section{Spinopelvic malalignment}

Spinal abnormalities have been identified as a risk factor for THA instability. Spinal abnormalities such as degenerative disease and spinal fusions result in reduced pelvic flexion. ${ }^{5}$ The pelvis has normal motion from standing to sitting which involves a pelvic posterior tilt arc of motion of 20 degrees and hip flexion ranging 55 to 70 degrees. The acetabular anteversion and inclination increases as the pelvis tilts posteriorly during sitting, and this facilitates the clearance of the femoral head and neck during hip flexion. The loss of this natural motion caused by stiffness of the spine results in compensatory increased hip motion which increases the risk of impingement and subsequent dislocation. ${ }^{22}$

\section{latrogenic}

IPD has been described in the setting of closed reduction of a hemiarthroplasty; this phenomenon was called the 'bottle opener' effect by Loubignac and Boissier. ${ }^{23}$ The proposed mechanism is that during closed reduction there is engagement of the polyethylene liner with the acetabular rim or in the setting of a dual mobility head on the acetabular cup. This is followed by dissociation of the inner bearing surface. This mechanism has been cited in reported early cases. ${ }^{2,15,21}$ To reduce the incidence of IPD, the reduction of dislocated DMC should not be attempted under conscious sedation and instead under general anaesthesia with muscle relaxation to prevent muscle contraction and subsequent rim impingement. ${ }^{14-16}$ In cases of dislocated DMC, care should be taken during the reduction, and it is also recommended to use intraoperative imaging to ensure both bearing surfaces are concentric after reduction. ${ }^{9}$ This described mechanism is in keeping with our presented case.

\section{Classification}

Philippot et al. proposed a classification which was mechanistic based on radiographic and intra-operative findings. Type 1 is due to wear of polyethylene retentive rim and the absence of arthrofibrosis and loosening. Type 2 is due to blocking of the larger polyethylene articulation resulting from arthrofibrosis or heterotopic ossification. Type 3 is due to loosening. ${ }^{2}$ This classification proposed three causes of intraprosthetic dislocation based on the respective aetiology. We propose a modification of this classification to include a type 4 iatrogenic.

\section{Conclusion}

DMC offers increased stability in THA. Our case study presents the 31 st reported case of early IPD as a complication of an attempted closed reduction of a DMC dislocation. The limited literature is insufficient to summarise the mechanisms of IPD. The diagnosis of IPD is an indication for revision surgery of the DMC. When utilising 
a DMC, care should be taken to mitigate against the known risk factors for IPD - notably all dual mobility dislocation should be reduced under general anaesthesia with muscle relaxant.

\section{Ethics statement}

The authors declare that this submission is in accordance with the principles laid down by the Responsible Research Publication Position Statements as developed at the 2nd World Conference on Research Integrity in Singapore, 2010.

The patient provided written informed consent for print and electronic publication of the case report.

\section{Declaration}

The authors declare authorship of this article and that they have followed sound scientific research practice. This research is original and does not transgress plagiarism policies.

\section{Author contributions}

AS: Data analysis, first draft preparation, manuscript preparation

KVJ: Data capture, data analysis

NS: Manuscript revision

LM: Manuscript revision

DVJ: Study conceptualisation, manuscript revision

\section{References}

1. Henawy AT, Abdel Badie A. Dual mobility total hip arthroplasty in hemiplegic patients. SICOT J. 2017;3:40. https://doi.org/10.1051/sicotj/2017024

2. Philippot R, Boyer B, Farizon F. Intraprosthetic dislocation: a specific complication of the dual-mobility system. Clin Orthop Relat Res. 2013;471(3):965-70. https://doi.org/910.1007/s11999-11012-12639-11992.

3. Plummer DR, Haughom BD, Della Valle CJ. Dual mobility in total hip arthroplasty. Orthop Clin North Am. 2014;45(1):1-8. https://doi.org/10.1016/j. ocl.2013.1008.1004.

4. Plummer DR, Christy JM, Sporer SM, Paprosky WG, Della Valle CJ. Dual-mobility articulations for patients at high risk for dislocation. J Arthroplasty. 2016;31(9 Suppl):131-35. https://doi.org/110.1016/j.arth.2016.1003.1021.

5. Rowan FE, Salvatore AJ, Lange JK, Westrich GH. Dual-mobility vs fixed-bearing total hip arthroplasty in patients under 55 years of age: a singleinstitution, matched-cohort analysis. J Arthroplasty. 2017;32(10):3076-81. https://doi.org/3010.1016/j.arth.2017.3005.3004.

6. Gwam C, Mistry J, Mohamed N, et al. Current epidemiology of revision total hip arthroplasty in the United States: national inpatient sample 2009 to 2013. J Arthroplasty. 2017;3(7):2088-92. https://doi.org/2010.1016/j. arth.2017.2002.2046.

7. Bozic K, Kurtz S, Lau E, et al. Epidemiology of revision total hip arthroplasty in the United States. J Bone Joint Surg Am. 2009;91(1):128-33.

8. Haynes J, Stambough J, Sassoon A, et al. Contemporary surgical indications and referral trends in revision total hip arthroplasty: a 10-year review. J Arthroplasty. 2016;31(3):622-25. https://doi.org/610.1016/j. arth.2015.1009.1026.

9. De Martino I, D'Apolito R, Waddell BS, et al. Early intraprosthetic dislocation in dual-mobility implants: a systematic review. Arthroplast Today. 2017;3(3):197202. https://doi.org/110.1016/j.artd.2016.1012.1002.

10. Sutter EG, McClellan TR, Attarian DE, et al. Outcomes of modular dual mobility acetabular components in revision total hip arthroplasty. J Arthroplasty. 2017;32(9S):S220-S224. https://doi.org/210.1016/j.arth.2017.1003.1035.

11. McArthur BA, Nam D, Cross MB, Westrich GH, Sculco TP. Dual-mobility acetabular components in total hip arthroplasty. Am J Orthop (Belle Mead NJ). 2013;42(10):473-78.

12. Hernigou P, Dubory A, Potage D, Roubineau F, Flouzat Lachaniette $\mathrm{CH}$. Dual-mobility arthroplasty failure: a rationale review of causes and technical considerations for revision. Int Orthop. 2017;41(3):481-90. https://doi. org/410.1007/s00264-00016-03328-00267.

13. Tabori-Jensen S, Hansen TB, Stilling M. Low dislocation rate of Saturne ${ }^{\circledR /}$ Avantage ${ }^{\circledR}$ dual-mobility THA after displaced femoral neck fracture: a cohort study of 966 hips with a minimum 1.6-year follow-up. Arch Orthop Trauma Surg. 2019;139(5):605-612. https://doi.org/610.1007/ s00402-00018-03093-00408.

14. Neri T, Boyer B, Batailler C, et al. Dual mobility cups for total hip arthroplasty: tips and tricks. SICOT J. 2020;6:17. https://doi.org/10.1051/sicotj/2020018.

15. Addona JL, Gu A, De Martino I, et al. High rate of early intraprosthetic dislocations of dual mobility implants: a single surgeon series of primary and revision total hip replacements. J Arthroplasty. 2019;34(11):2793-98. https:// doi.org/2710.1016/j.arth.2019.2706.2003.
16. Rotini M, Cianforlini M, Aucone D, Pacetti E, Politano R. latrogenic intraprosthetic dislocation after closed reduction of dual mobility total hip arthroplasty: Report of two cases. Int J Surg Case Rep. 2020;71:225-29. https://doi.org/210.1016/j.ijscr.2020.1004.1085.

17. McKee G, Watson-Farrar J. Replacement of arthritic hips by the McKee-Farrar prosthesis. J Bone Joint Surg Br. 1966;48(2):245-59.

18. Terrier A, Latypova A, Guillemin M, Parvex V, Guyen O. Dual mobility cups provide biomechanical advantages in situations at risk for dislocation: a finite element analysis. Int Orthop. 2017;41(3):551-56. https://doi.org/510.1007/ s00264-00016-03368-z.

19. Chouteau J, Rollier JC, Bonnin MP, et al. Absence of instabilities and intra-prosthetic dislocations at 7 to 11 years following THA using a fourthgeneration cementless dual mobility acetabular cup. J Exp Orthop. 2020;7(1):51. https://doi.org/10.1186/s40634-40020-00265-40633.

20. You D, Sepehri A, Kooner S, et al. Outcomes of total hip arthroplasty using dual mobility components in patients with a femoral neck fracture. Bone Joint J. 2020;102-B(7):811-21. https://doi.org/810.1302/0301-1620x.1102b1307. bjj-2019-1486.r1301.

21. De Martino I, D'Apolito R, Waddell BS, et al. Response to letter to the editor on 'Early intraprosthetic dislocation in dual-mobility implants: a systematic review'. Arthroplast Today. 2018;4(1):133-34. https://doi.org/110.1016/j. artd.2017.1011.1005.

22. Hiroyuki I, Dorr L, Transolini N, et al. Spine-pelvis-hip relationship in the functioning of a total hip replacement. J Bone Joint Surg Am. 2018;100A(18):1606-15. https://doi.org/1610.2106/jbjs.1617.00403.

23. Loubignac F, Boissier F. [Cup dissociation after reduction of a dislocated hip hemiarthroplasty]. Rev Chir Orthop Reparatrice Appar Mot. 1997;83(5):469-72. PMID: 9452801 